\title{
Topological defects in cholesteric liquid crystals induced by monolayer domains with orientational chirality
}

\author{
Núria Petit-Garrido, ${ }^{a, b}$ Rahul P. Trivedi, ${ }^{b}$ Francesc Sagués, ${ }^{a}$ Jordi Ignés-Mullol, ${ }^{* a}$ and \\ Ivan I. Smalyukh*b,c
}

Received (in $X X X, X X X)$ Xth $X X X X X X X X X 20 X X$, Accepted Xth $X X X X X X X X X 20 X X$ DOI: $10.1039 / \mathbf{b 0 0 0 0 0 0 x}$

Unless stabilized by colloids or confinement with well-defined boundary conditions, defects in liquid crystals remain elusive short-lived objects that tend to annihilate with time to minimize the medium's free energy. In this work we use multimodal threedimensional imaging to visualize cholesteric director structures to show that selfassembled chiral molecular monolayer domains can stabilize topologically constrained defect configurations when in contact with a cholesteric liquid crystal. The cholesteric liquid crystal, having features of both coarse-grained lamellar and nematic liquid crystal with chiral symmetry breaking, allows us to explore the interplay of chirality and implications of layering on the formed defects and director configurations. 


\section{Cite this: DOI: 10.1039/c0xx00000x}

\section{Wednesday, 30 January 2019}

\section{Introduction}

Orientational or partial translational order is present in many soft matter systems, including liquid crystals (LCs) ${ }^{1}$, lipid 5 monolayers $^{2}$ and biomembranes ${ }^{3}$. Under confined geometries, topological incompatibilities with boundary conditions lead to frustration in the orientational order and to the proliferation of topological defects ${ }^{4}$. The ability to control topological defects in LCs is crucial to the development of new devices, sensors, and 10 materials ${ }^{5-8}$. Recent research has focused on devising novel types of self-assembled LC-based functional materials by understanding the defects and the healing of the disorder introduced by confining surfaces ${ }^{9}$, contact with embedded colloidal particles ${ }^{8,10-12}$, or confinement in spherical or shell-like 15 emulsions $^{13}$.

Controlled generation of multistable textures that include disclination lines has been achieved by patterning boundary conditions on surfaces using micro-engineered polymer layers, either by means of mechanical scribing ${ }^{14,}{ }^{15}$, through ${ }_{20}$ photopolymerization methods ${ }^{16}$, or using standard photolithography techniques ${ }^{17}$. In contrast to these artificial patterning methods, complex $3 \mathrm{D}$ configurations can be imprinted in LCs through interaction with self-assembled surfactant monolayers formed by means of the Langmuir-Blodgett (LB)

25 technique $^{18,}$ 19. In a recent work $^{20}$, we have shown that the patterns of molecular alignment present in Langmuir monolayers of an azobenzene surfactant can be transferred into a nematic LC by confining the mesogen between a glass plate coated with the LB film and a complementary plate treated for planar alignment, 30 the latter of which sets the homogeneous far field LC orientation

(Fig. 1). When spread at the air/water interface, these surfactant molecules with polar symmetry spontaneously organize into submillimeter circular disk-like domains rich in the elongated trans isomer surrounded by a disordered matrix rich in the bent 35 cis isomer. Inside the disks, hydrophobic chains are locally tilted tangentially to the circular boundary, either with clockwise $(\mathrm{CW})$ or counter-clockwise $(\mathrm{CCW})$ orientational chirality with equal probability of either handedness. These domains feature a pure bend texture that includes a central point singularity of 40 topological charge $+1 .^{21}$ When in contact with the LB film, the LC director $\mathbf{n}(\mathbf{r})$ follows the local in-plane orientation of the surfactant monolayer (Fig. 1) ${ }^{18,20,22}$. The anchoring angle with respect to the surface normal is similar throughout the LB film $\left(35^{\circ}-45^{\circ}\right)$, but outside the domains surfactant molecules are 45 disordered, and the LC director adopts an in-plane alignment set by the complementary plate. Matching of the boundary conditions on the circular disk and on the planar far-field alignment leads to a defect healing scenario that results in the formation of either single twist-escaped or double singular arch${ }_{50}$ like disclinations in $\mathbf{n}(\mathbf{r})$. In both cases, the disclinations are pinned on point defects (boojums) at the LB-coated-surface: a $\mathrm{s}=+1$ boojum at the domain center and either one $\mathrm{s}=-1$ (single arch) or two $s=-1 / 2$ (double arch) boojums at the boundary. The final configuration depends on a combination of temperature 55 cycling, chirality of the underlying LB domain, and nematic LC flow direction during cell filling. Across the cell gap an inhomogeneous twist in $\mathbf{n}(\mathbf{r})$ is formed, with a magnitude and sign that minimizes the distortion required to satisfy the mixed boundary conditions. Due to the symmetry breaking introduced 60 by cell filling, twist is maximum (half a turn) in the region where surfactant molecules are antiparallel to the flow (twist-escaped disclination) while it vanishes across the domain, where surfactant molecules are parallel to nematic flow (Fig. 1). On the other hand, the sign of the twist distortion is opposite on either 65 side of the disclination. In fact, $\mathbf{n}(\mathbf{r})$ adopts enantiomorphic (mirror-symmetric) configurations in contact with monolayer domains of either orientational handedness. Both these two configurations change to a double arch texture upon transition cycling into the isotropic phase and back to nematic. In the 70 experiments with nematic LCs, domains with twist distortions of opposite handedness coexist naturally because of their comparable energy costs.

The above defect healing scenario might be significantly altered by changing the lateral order of the underlying monolayer or the 75 symmetry in the mesogen. In particular, the incorporation of a chiral dopant will lead to the formation of a cholesteric LC, which has features of both coarse-grained lamellar and nematic liquid crystal with chiral symmetry breaking ${ }^{4}$. In contact with the patterned LB films, this system is expected to exhibit an 80 interesting interplay between chirality in three-dimensional and two-dimensional ordered systems.

In the present work we study cholesteric LC cells built using the mixed boundary conditions established by combining an LB85 coated plate (having domains with orientational chirality) and a complementary plate defining a planar far field alignment. The twist imposed by the boundary conditions results in regions with different elastic energy, depending on whether the local handedness has the same or opposite sign as that of the intrinsic 90 pitch of the chiral mesogen. We employ a combination of twodimensional and three-dimensional polarizing microscopy techniques, and reveal that the single arch and double arch disclinations obtained in nematic LC cells transform into different strength (Burgers vector) edge dislocations, characteristic of ${ }_{95}$ cholesteric LC and other lamellar materials. We begin by studying the continuous transformation from nematic to cholesteric LC cells by employing a mixture of mesogens with a strong temperature dependence of the cholesteric pitch around a compensation point. 

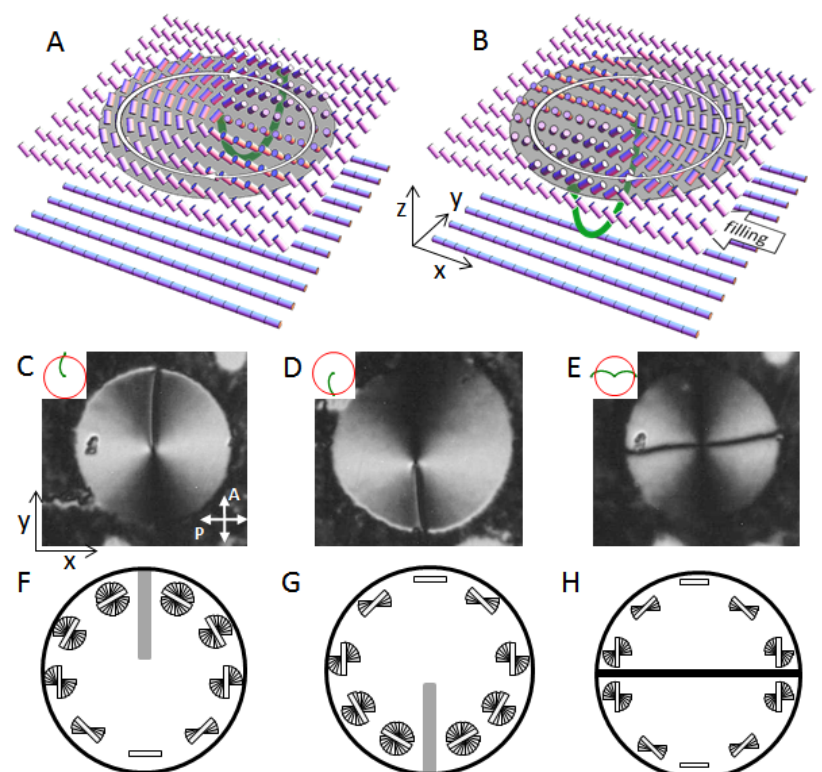

G
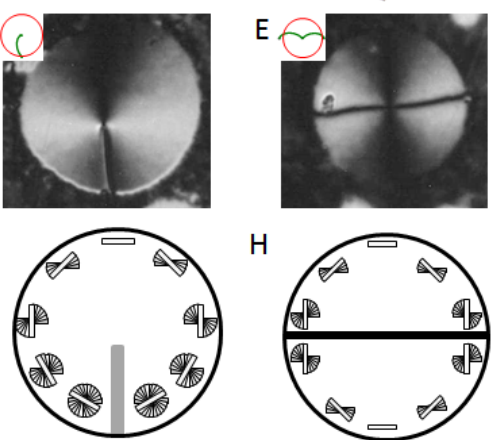

Figure 1. The structure of hybrid NLC cells. Surfactant molecules on the LB-coated top plate organize in submillimeter circular domains with $\mathrm{CW}$ (A) or CCW (B) orientational handedness. The local in-plane order 5 propagates to the mesogen as illustrated by the cylinder plot. Horizontal far-field is set by the bottom plate with planar alignment. (C-E) Optical micrographs of representative NLC microdomains in the three observed configurations. The width of each frame is $120 \mu \mathrm{m}$. (F-H) Corresponding distribution of local twist across the cells. Upon cell filling, a single-arch 10 twist-scaped disclination forms perpendicular to the mesogen flow-filling direction, developing in different positions for $\mathrm{CW}(\mathrm{A}, \mathrm{C})$ and $\mathrm{CCW}(\mathrm{B}$, D) handedness of the underlying domain, resulting in an inhomogeneous twist $(\mathrm{F}, \mathrm{G})$. When transited to the isotropic and back to the nematic phase, a horizontal double-arch singular disclination develops (E and $\mathrm{H})$.

\section{${ }_{15}$ Materials and methods}

\section{Sample Preparation}

Glass substrates of an area of a few square centimeters were cleaned with fresh piranha solution composed of $30 \%$ aqueous $\mathrm{H}_{2} \mathrm{O}_{2}$ and $70 \%$ of concentrated $\mathrm{H}_{2} \mathrm{SO}_{4}$. The cleaning solution was $20 \mathrm{kept}$ at $70^{\circ} \mathrm{C}$ in a water bath for $1 \mathrm{~h}$. The plates were subsequently cooled, rinsed with abundant Milli-Q water and dried thoroughly with a stream of nitrogen. One of the plates was patterned by LB transfer of monolayers of the surfactant 4-[4-[4-octylphenyl/azo]phenoxy]butanoic acid $(8 \mathrm{Az} 3 \mathrm{COOH})$, previously self-assembled 25 at the air/water interface in the form of isolated islands at low lateral pressure $(<1 \mathrm{mN} / \mathrm{m})$, as described previously ${ }^{20}$. This process was carried out using either a Nima 600 Series LB instrument or a custom-made system at room temperature and using a dipper speed of $1 \mathrm{~mm} / \mathrm{min}$.

30

The complementary plate of the assembled LC cells imposes uniform planar boundary conditions away from the LB-coated plate. This is achieved by means of a rubbed, spin-coated layer of polyimide (PI-2555, HD MicroSystems) prepared on the 35 complementary plate. For the experiments with compensated mixtures, cells were filled by capillarity with a mixture of $8 \mathrm{OCB}$ (Synthon Chemicals) and the chiral molecule Cholesteryl Chloride (Sigma), both used as received, in proportion 1:1 by weight. This mixture features a strongly temperature-dependent 40 cholesteric pitch with a compensation temperature $\mathrm{T}_{\mathrm{c}} \sim 56-60^{\circ} \mathrm{C}$
23 and a clearing point at $66^{\circ} \mathrm{C}$. We have measured the cholesteric pitch as a function of temperature by analyzing the Frank-Pryce textures in LC-in-water emulsions with planar anchoring, stabilized with poly-vinyl alcohol ${ }^{13}$. A small volume of ${ }_{45}$ chloroform (Baker p.a.) was added to the two solid compounds to dissolve them and facilitate their mixture. The solution was subsequently heated to the isotropic phase in an ultrasound bath to properly mix the two components and let the chloroform evaporate. Both the empty cells and the LC were held at a 50 temperature close to the compensation value prior to cell filling. Strips of $13 \mu \mathrm{m}$ Mylar film (GoodFellow) were used as spacers for these cells. For fluorescence confocal polarizing microscopy (FCPM) and two-photon excitation fluorescence polarizing microscopy (2PEFPM) studies, we used as a chiral nematic host 55 a low-birefringence LC mixture (ZLI-2806 doped with $1.5 \% \mathrm{w} / \mathrm{w}$ of chiral agent CB15, EM Chemicals) doped with about 0.01 wt. $\%$ of the fluorescent dye n,n'-bis(2,5-di-tert-butylphenyl) 3,4,9,10-perylenedicarb- oximide (BTBP, Aldrich). Threephoton excitation fluorescence polarizing microscopy (3PEFPM) 60 requires no dye dopant, since self-fluorescence of the mesogen is directly excited by means of multiphoton absorption processes. Monodisperse silica beads (diameter 10, 15, $30 \mu \mathrm{m}$ ) placed between the plates acted as spacers, to obtain various desired values of cell-thickness.

65

\section{Polarized Light Microscopy}

Samples were held at a controlled temperature using a custommade computer-controlled temperature oven, and observed using a Nikon Eclipse 50i polarizing microscope. A color digital 70 camera (AVT Marlin F-131C) was used for image acquisition. The public domain software Image $^{24}$ was used for image processing.

\section{Three dimensional Microscopy}

FCPM, 2PEFPM, and 3PEFPM images were obtained by use of 75 an integrated multimodal imaging setup built around an Olympus IX-81 inverted microscope. For FCPM an Olympus Fluoview FV300 confocal unit is employed, with a $488 \mathrm{~nm}$ Ar ion laser to excite the BTBP dopant dye fluorescence. For multiphoton excitation microscopy we used the same laser scanning device 80 without confocal unit. Laser excitation was performed with a femtosecond Ti:sapphire oscillator (140 fs, $80 \mathrm{MHz}$, Chameleon Ultra-II, Coherent). This pulsed laser allows for wavelength tunability within $680-1080 \mathrm{~nm}$. Two-photon excitation in 2PEFPM was performed with a $980 \mathrm{~nm}$ wavelength and by ${ }_{85}$ placing a band pass filter transmitting between 510 and $560 \mathrm{~nm}$ in front of the detector. For 3PEFPM, excitation was performed at $860 \mathrm{~nm}$ and a detector band pass filter transmitting between 387 and $447 \mathrm{~nm}$. Excitation intensity was kept minimal in all cases to avoid altering the photosensitive LB films and the director 90 structures in the LC. Although the precursor monolayer has been proven to be photosensitive at the air/water interface under irradiation at similar wavelengths ${ }^{25}$, steric hindrance inside the ordered domains and slow diffusion of surfactant molecules on the LB film allows observations to be performed without 95 detectable alterations in the LC patterns

Imaging was performed with colinear polarization directions for excitation and detected fluorescence light. The transition dipole 
moments of absorption and emission of the anisotropic BTBP dye molecules follow the liquid crystal director $\mathbf{n}(\mathbf{r})$. Therefore, in the case of polarized epidetection of fluorescence signals, fluorescence intensity in the images scales as $\cos ^{4} \theta$ in FCPM, as ${ }_{5} \cos ^{6} \theta$ in 2PEFPM, and as $\cos ^{8} \theta$ in 3PEFPM (for polarized fluorescence detection), where $\theta$ is the angle between the polarization of probing light and $\mathbf{n}(\mathbf{r})$; note that unpolarized detection in these techniques is also often used, in which case the detected signals scale as $\cos ^{2} \theta$ in FCPM, as $\cos ^{4} \theta$ in 2PEFPM, 10 and as

$\cos ^{4} \theta$ for

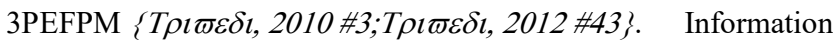
about the spatial variation in the orientation of $\mathbf{n}(\mathbf{r})$ is extracted by comparing images acquired for different polarization directions of probing light. Using 2PEFPM and 3PEFPM is 15 advantageous with respect to FCPM, particularly in thick samples, given their improved sensitivity and deeper penetration into the sample. ${ }^{26}$ Image J and Mathematica were used for data analysis and presentation.

\section{${ }_{20}$ Results and Discussion}

\section{Introduction of cholesteric order in hybrid nematic cells}

In order to gradually introduce cholesteric lamellar-like order in the nematic system we employ the mixture $8 \mathrm{OCB} / \mathrm{CC}(1: 1)$ that features a cholesteric phase with a compensation temperature $T_{c}$ ${ }_{25} \sim 56{ }^{\circ} \mathrm{C}-60{ }^{\circ} \mathrm{C}$ at which the pitch diverges (the material becomes nematic), and a strong temperature-dependence of the pitch above and below this temperature ( $p$ is right-handed above $T_{c}$ and lefthanded below $\left.\mathrm{T}_{\mathrm{c}}\right)^{23}$. Typical values are $|p|=45 \mu \mathrm{m}$ for $\left|\mathrm{T}-\mathrm{T}_{\mathrm{c}}\right|=$ $1.0^{\circ} \mathrm{C}$. Following previously described procedures ${ }^{20}$, we prepared 30 nematic samples on hybrid cells featuring the single arch configuration (single twist-escaped disclination) described above. The liquid crystal is introduced in the hybrid cell by capillarity at a temperature $T \sim T_{c}$. In contact with disks, hybrid boundary conditions impose a non-uniform twist across the liquid crystal 35 cell gap, as explained above (Fig. 1). In the nematic phase, and for the example presented in Figure 2 where the liquid crystal organizes over an LB domain with $\mathrm{CW}$ azimuthal orientation, twist is right handed $(\mathrm{RH})$ to the right of the disclination and left handed $(\mathrm{LH})$ to the left (Fig. 2A, B). When the temperature 40 departs from $T_{c}$, the CLC intrinsic pitch becomes finite and with a well-defined handedness. As a consequence, the elastic energy density will be different on both sides of the disclination, since the twist imposed by boundary conditions will have the same sign as the intrinsic LC handedness on one side and the opposite sign 45 on the other side. This drives the expansion of the region in which the twist across the gap has the same sign as the CLC pitch. For instance, in the example in Fig. $2 \mathrm{C}$ and $\mathrm{E}$, the archshaped disclination bends towards the right (region with lefthanded twist grows) for $\mathrm{T}<\mathrm{T}_{\mathrm{c}}$ (CLC pitch is left handed). Notice 50 that the disclination remains pinned to the same pair of boojum defects on the LB-coated plate at all times, so its length increases as $\mathrm{T}$ departs from $\mathrm{T}_{\mathrm{c}}$. The remarkable pinning strength of these boojums was already reported earlier ${ }^{20}$ by showing that they remain unaltered when pulling the disclination lines by means of 55 optical tweezers, and has been demonstrated in recent experiments where the configuration of NLC samples is perturbed by means of an electric field ${ }^{27}$. This is in contrast to similar experiments performed in the so-called theta-cell ${ }^{28,} 29$ constructed by combining a circularly rubbed polyimide-treated ${ }_{60}$ glass plate with one that is unidirectionally rubbed. In that case, pinning on the plates was not as strong as it is on the LB domains, and the disclination was free to rotate as the CLC pitch changed. This resulted in a characteristic equilibrium angle of the disclination line as the boojum on the periphery circumnavigated. ${ }_{65}$ This angle was directly related to the pitch, as elastic energy in the untwisted and overtwisted regions on both sides of the disclination balance ${ }^{29}$. Although a similar equilibrium configuration as a function of the temperature-dependent CLC pitch exist in the present case, the complex three-dimensional 70 nature of $\mathbf{n}(\mathbf{r})$ makes a similar analytical study impractical.

Further departure from $T_{c}$ leads to a continued extension of the disclination, which evolves parallel to the boundary of the underlying circular domain. We notice that the disclination 75 appears to be twisted about a point singularity that nucleates on the $\mathrm{s}=-1$ boojum and separates from it as the temperature departs from $\mathrm{T}_{\mathrm{c}}$ (arrow in Fig. 2E).

When the value of $p$ approaches $d / 2$, regions with half turn twist 80 can nucleate and grow anywhere in the cholesteric material in this confined geometry. In fact, the rotating disclination acts as the nucleation site of such regions by expanding beyond the limits of the circular domain and into the originally untwisted regions (see video "moviel.mov" in the Supplementary Information for 85 changes in a CLC cell in a region with several circular domains of either chirality, when the temperature is lowered from $T_{c}$ down to $\mathrm{T}_{\mathrm{c}}-7^{\circ} \mathrm{C}$ ). In fact, by increasing the range of the temperature changes we observe the continuous nucleation and propagation of dislocations, and we see interaction between the original island90 induced defect and the line defects in the CLC bulk (Fig. 2). 

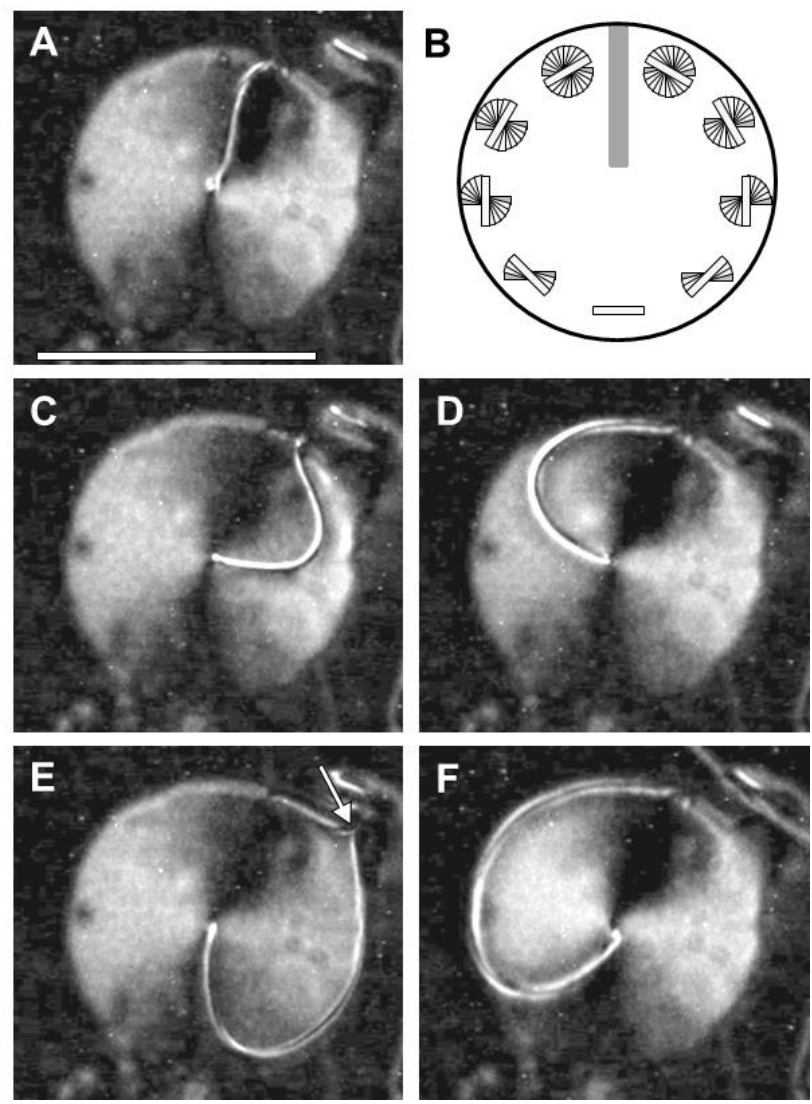

Figure 2. Optical micrographs of a circular domain inside a compensated CLC cell for different values of the T-controlled cholesteric pitch. The cell gap is $12 \mu \mathrm{m}$. The line segment in (A) is $100 \mu \mathrm{m}$ long. Anchoring 5 conditions are planar in the horizontal direction at the bottom plate and tangential to the circular boundary on the top LB-coated plate. (A) At the compensation temperature, $\mathrm{T}_{\mathrm{c}} \sim 56.1{ }^{\circ} \mathrm{C}$, the hybrid boundary conditions impose a non-uniform twist across the cell gap, as sketched in (B). This results in a twist-escaped disclination line that spans in the nematic 10 medium along a radius orthogonal to the flow-filling direction. Below $(\mathrm{C}$, E) and above $(D, F) T_{c}$, the pitch becomes finite. A point defect emerges from the boundary boojum, as indicated by the arrow (E). Temperature and corresponding approximated pitch conditions are, respectively, $\mathrm{T}_{\mathrm{c}^{-}}$ $0.55^{\circ} \mathrm{C},-84 \mu \mathrm{m}(\mathrm{C}) ; \mathrm{T}_{\mathrm{c}}+0.55^{\circ} \mathrm{C},+84 \mu \mathrm{m}(\mathrm{D}) ; \mathrm{T}_{\mathrm{c}}-0.95^{\circ} \mathrm{C},-48 \mu \mathrm{m}(\mathrm{E}) ; \mathrm{T}_{\mathrm{c}^{-}}$ $150.95^{\circ} \mathrm{C},+48 \mu \mathrm{m}(\mathrm{F})$.

The behaviour of the single arch configuration is completely different from that of the double arch one, the latter being obtained upon cycling the CLC sample into the isotropic and back into the mesophase. ${ }^{20}$ The double-arch disclinations remain 20 unaltered by changes in the cholesteric pitch or by the transit of oily streaks, indicating that the configuration of the director field around the double-arch disclination is different from the defect structures spontaneously formed in CLC (see video "movie2.mov" in the Supplementary Information corresponding 25 to a region near a single domain with the double-arch configuration, when the temperature is raised from $\mathrm{T}_{\mathrm{c}}-7^{\circ} \mathrm{C}$ up to $\mathrm{T}_{\mathrm{c}}$ ). In spite of the apparent similarity, the latter are therefore different from the singular disclinations reported earlier by Suh et al. ${ }^{29}$ with macroscopic theta cells, which always generated a 30 double disclination that rotates when changing the cholesteric pitch.

In order to elucidate the three-dimensional configuration of the CLC director in the vicinity of the linear defect structures
35 generated by our twisted boundary conditions, we resort to threedimensional microscopy techniques using a constant pitch CLC mixture, which we report in the next section.
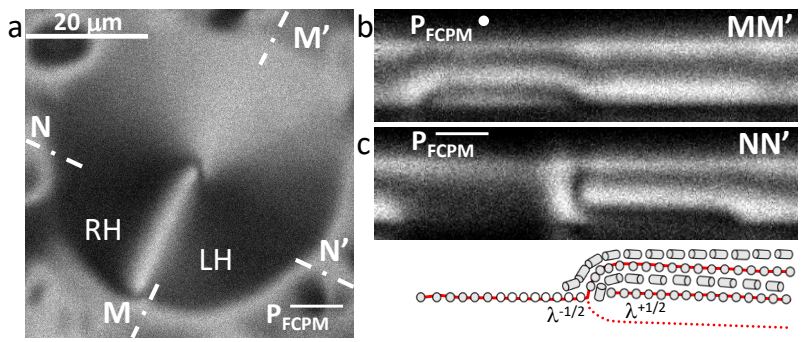

Figure 3. In plane (a) and vertical (b, c) FCPM cross sections over a $\mathrm{CCW}$ circular domain in the vicinity of the LB-coated plate after filling the cell with the CLC from right to left. The sample is $6 \mu \mathrm{m}$ thick. A sketch of the director field in the NN' cross section is included below (c).

45 The handedness of the twist imposed by the boundary conditions on both sides of the disclination is labelled in panel (a) and competes or augments the chirality of the LC.

a
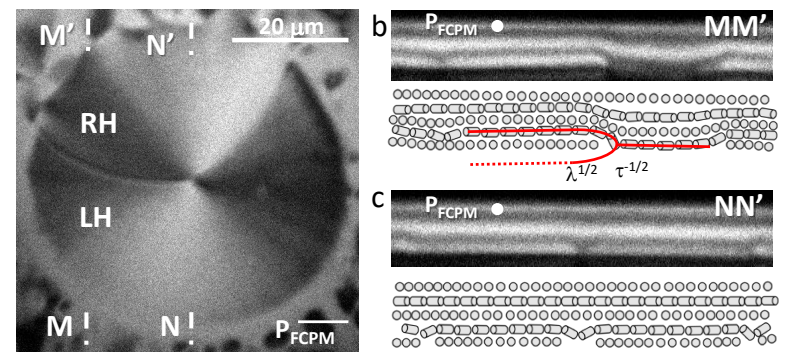

Figure 4. In plane (a) and vertical (b, c) FCPM cross sections over a 50 $\mathrm{CCW}$ circular domain in the vicinity of the LB-coated plate. The sample has been temperature cycled to the isotropic and back into the cholesteric phase. The cell gap is $10 \mu \mathrm{m}$. A sketch of the director field in the vertical cross section is included $(b, c)$. The handedness of the twist imposed by the boundary conditions on both sides of one of the disclinations is 55 labelled in panel (a) and competes or augments the chirality of the LC.

\section{Three-dimensional configuration of the cholesteric director}

To carry out these observations, we have prepared cells with a mixture based on the nematic host ZLI-2806 doped with about $1.5 \% \mathrm{w} / \mathrm{w}$ of CB15, giving a right-handed CLC phase with pitch ${ }_{60} p=8.5 \mu \mathrm{m}$ (see below). In Figure 3 we present FCPM data for a sample with thickness $d=6 \mu \mathrm{m}$ filled with the CLC from right to left. An in-plane cross-section (Fig. 3.a) reveals the presence of a linear defect structure anchored to surface defects at two locations, analogous to the "single-arch" configuration observed ${ }_{65}$ with nematic $\mathrm{LC}^{20}$. A vertical section across the line defect (NN' in Fig. 3) reveals that the CLC director is partially untwisted to the left of the line (its twist is roughly one quarter of a turn), where the twist imposed by the boundary conditions has the same handedness as the material pitch $(\mathrm{RH})$, and the CLC director is 70 over-twisted as compared to the equilibrium pitch (its twist is roughly three-quarters of a turn) when the geometrical and the material twist have opposite signs. Notice that, since the cell thickness is about $3 / 4 p$, one would expect to realize a CLC director twist of half a turn across the cell gap in the planar far 75 field. A longitudinal section along the line defect (MM') shows 
that the director twist across the cell gap in the region with no geometrical twist has indeed the value expected given the ratio $p / d$. The reconstruction of the CLC director corresponding to the NN' FCPM cross section in Fig. 3c suggests that the boundary 5 conditions imposed by the submillimeter circular domains induce the formation of an edge dislocation in the cholesteric, which is split into a pair $\lambda^{-1 / 2} \lambda^{+1 / 2}$ of non-singular disclinations ${ }^{30}$ nearby the LB surface.

In Figure 4 we report FPCM data for a sample with thickness $d=$ ${ }_{10} 10 \mu \mathrm{m}$ that has been transitioned to the isotropic and back to the cholesteric. The in-plane cross section reveals two linear defects analogous to the "double-arch" configuration reported for nematic $\mathrm{LC}^{20}$. In Figure 4, the cell gap is slightly larger than the cholesteric pitch, so a full turn of the CLC director is expected in 15 the regions with uniform planar boundary conditions. Vertical cross sections across the line defect (Fig. 4b) reveal a partial untwist of the CLC director (roughly one quarter of a turn) in the region where the geometrical pitch of a nematic LC would have the same handedness as the dopant (RH), while the CLC director 20 appears to be unaffected by geometrical boundary conditions that oppose the material handedness (LH). A reconstruction of the CLC director corresponding to the MM' FCPM cross section (Fig. 4b) suggests that the CLC director develops two edge dislocations, one corresponding to each of the twin arches that 25 formed in nematic samples. The core of such a dislocation is split into two disclinations of type $\tau^{-1 / 2} \lambda^{+1 / 2}$, attached to the LB surface.

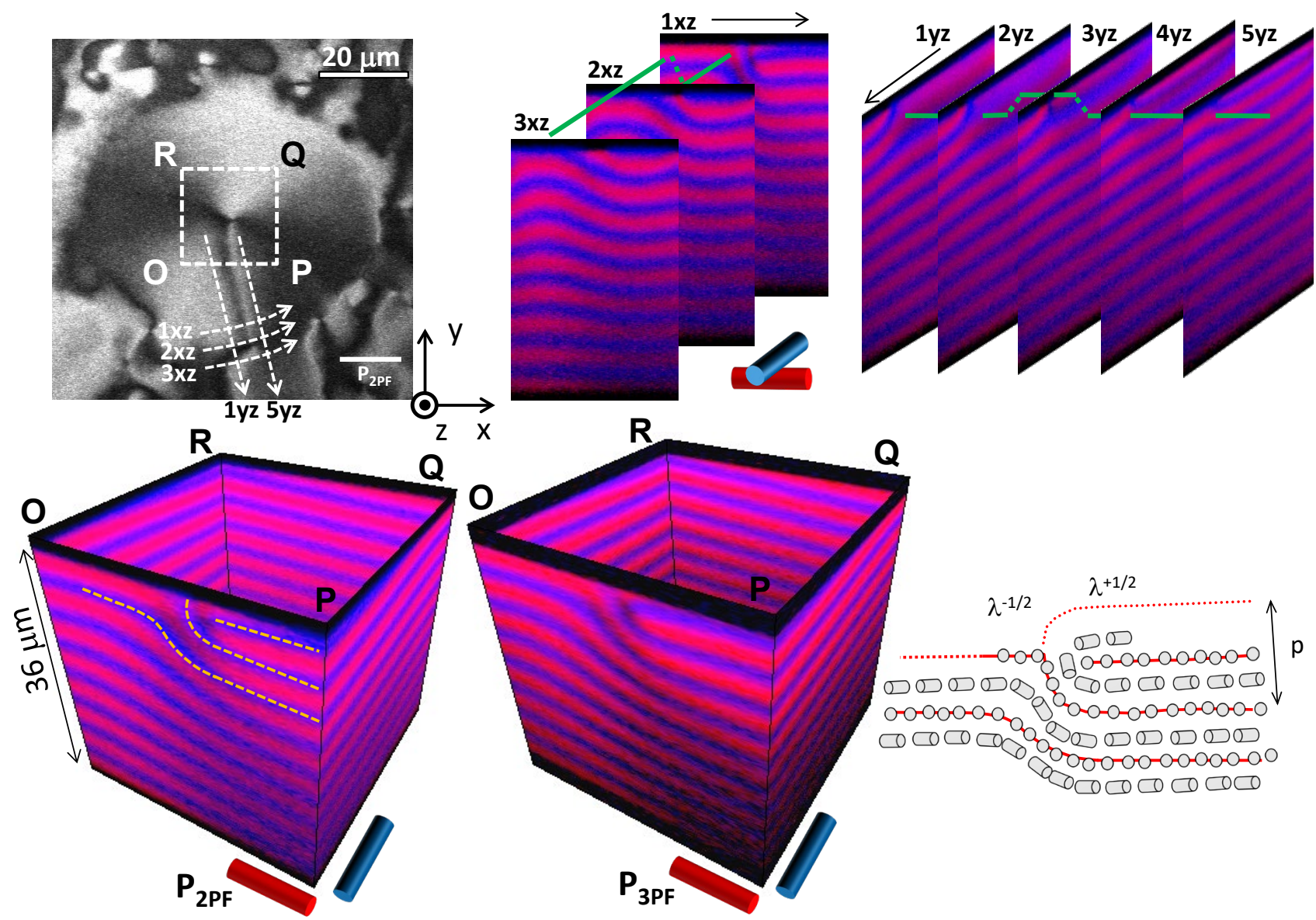

Figure 5. In plane and different vertical 2 PEFPM and 3PEFPM cross sections in the vicinity of a circular domain in the LB-coated top plate, after filling 35 the cell with the CLC from right to left, parallel to the far-field orientation imposed by the buffed poly-imide layer on the bottom complementary plate. Surfactant molecules on the surface domain are ordered with CCW handedness. Vertical cross-sections across the paths indicated in (a) are presented in different panels. Data for two orthogonal polarizations are combined by means of color-coding, as indicated. A sketch of the director field in the OP XZcross section is included. For comparison, both the 2PEFPM and 3PEFPM OPQR vertical cross-sections are presented.

In our earlier studies with nematic liquid crystals ${ }^{20}$, we showed 40 that the distortion in $\mathbf{n}(\mathbf{r})$ in the case of the single-arch twistescaped disclination had a penetration depth that scaled with sample thickness below $10 \mu \mathrm{m}$, and it reached an asymptotic value of $10-15 \mu \mathrm{m}$ for thick samples (up to $60 \mu \mathrm{m})^{20}$. In the case of CLC, there is an additional length-scale, the cholesteric pitch,
45 which should play an important role in confining the defect healing into a distance of the order of $p$. We have thus analysed the configuration of thicker cells $(p / d>>1)$, for which multiphoton microscopy techniques are more suitable, as they offer increased penetration depths ${ }^{26}$.

${ }_{50}$ In Figure 5, we present the 2PEFPM observations for a $36 \mu \mathrm{m}$ 
thick CLC cell in the two-boojum (single-arch) configuration in the vicinity of a LB domain. For comparison, data for 3PEFPM has also been included. We observe that this technique provides similar resolution by directly exciting the fluorescence of the 5 mesogen, without the need for a dopant. The data is obtained in the presence of a CCW domain in the LB-coated plate after the cell is filled right-to-left. Cross section data include two orthogonal polarizer configurations, and are combined in the presented color coded images. These are RGB color images, 8 bit 10 per channel, with the $G$ channel set to zero and the $R$ and $B$ channels incorporating data for each of the complementary (orthogonal) polarizer configurations, as indicated in each panel. While deep red regions correspond to $\mathbf{n}(\mathbf{r})$ oriented roughly along the $\mathrm{X}$ axis and deep blue regions correspond to $\mathbf{n}(\mathbf{r})$ oriented 15 roughly along the $\mathrm{Y}$ axis, intermediate orientations appear with a purple hue, as both the $\mathrm{R}$ and $\mathrm{B}$ channels are nonzero. The vertical cross section across the linear defect $(\mathrm{OP})$ reveals that this is indeed an edge dislocation of Burgers vector $b=p(\mathrm{p} \sim$ $8.5 \mu \mathrm{m}$, as estimated from the cross section images) attached to 20 the LB-coated surface. The core of the dislocation, which is attached to the LB-coated plate, is split into a pair $\lambda^{-1 / 2} \lambda^{+1 / 2}$ of non-singular disclinations. A cross section along the edge dislocation (3yz) confirms that the latter is pinned on two boojums, one at the center and the other on the periphery of the ${ }_{25}$ LB domain, consistently with the fact that the topologically stable dislocations cannot end in the bulk of the liquid crystal. Near the center of the domain, the edge dislocation is connected to the surface by $a b=p$ screw dislocation. The latter is originated by $\mathbf{n}(\mathbf{r})$ following the azimuth of the aliphatic chains on the LB film, 30 which perform a full rotation around the center of the circular domain. This generates a $\chi=1$ wedge disclination that propagates as a screw dislocation into the bulk CLC. The vertical cross section along the circuit OPQRO in Fig. 5 allows visualizing how the cholesteric layers organize around the screw 35 dislocation. The edge dislocation is not present outside of the domain, so it must glide in order to appear as we enter the domain. Dislocation gliding involves the formation of kinks ${ }^{30}$, and these are indeed observed at the periphery of the LB domain, where the edge dislocation present inside the domain is brought 40 back onto the surface by a kink $(1 \mathrm{xz}-3 \mathrm{xz})$. Along the boundary $(1 \mathrm{yz}-5 \mathrm{yz})$, the pinning of the edge dislocation results in a kinkantikink pair.
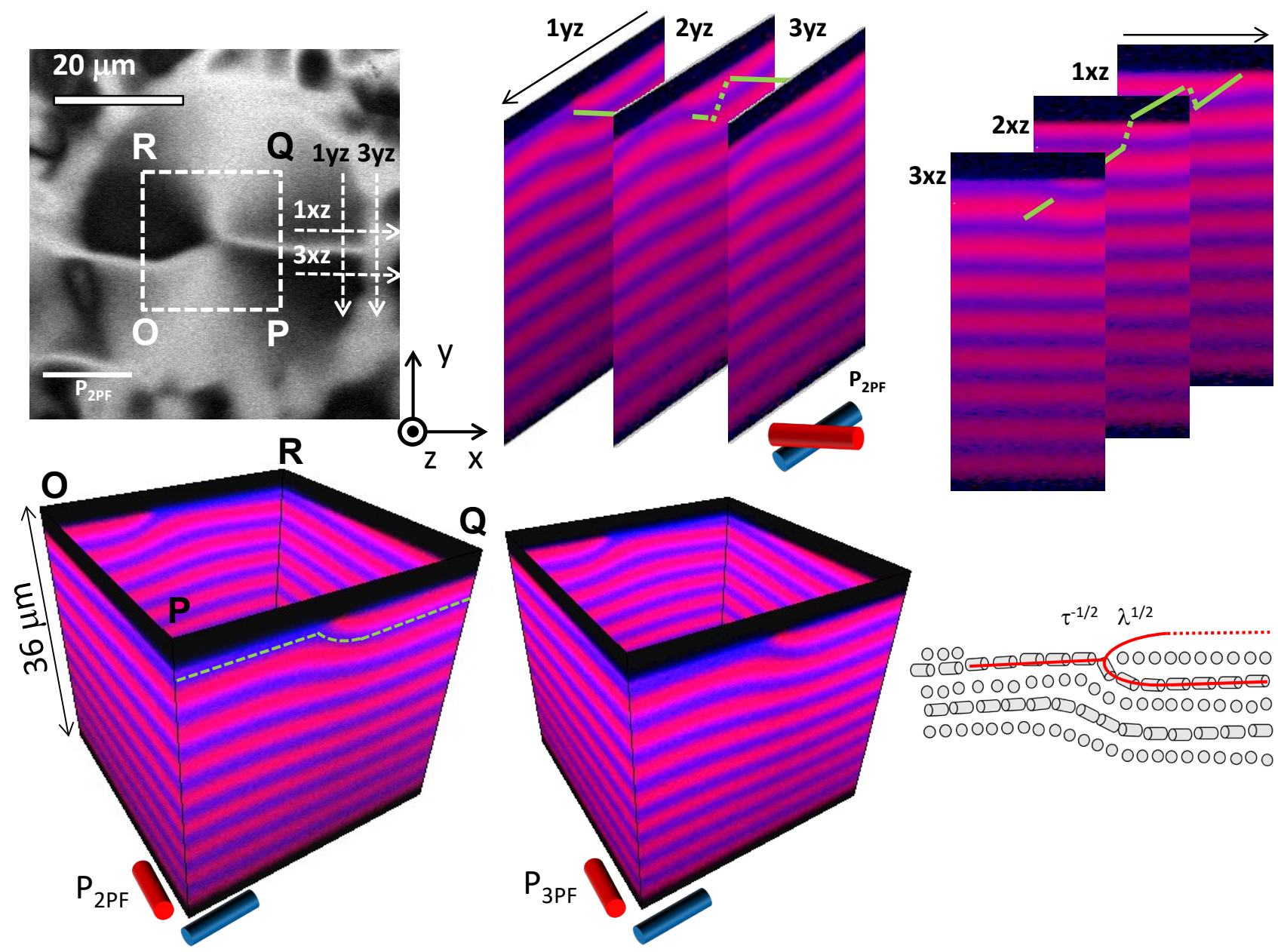

45 Figure 6. In plane and different vertical 2PEFPM cross sections for the same sample presented in Figure 5 after a transition into the isotropic and back to the CLC phase. Vertical cross-sections across the paths indicated in (a) are presented in different panels. Data for two orthogonal polarizations are combined by means of color-coding, as indicated. A sketch of the director field in the PQ YZ cross section is included. For comparison, both the 2PEFPM and 3PEFPM OPQR vertical cross-sections are presented. 
By comparing data in Figures 3 and 5, we observe that the archshaped edge dislocation is pinned on two boojum on the LB film, and penetrates into the bulk CLC for a length $p / 2$, as long as this 5 distance is smaller than the cell gap. This is different from the observations in nematic cells, where the penetration length of the corresponding twist-escaped disclination scaled with sample thickness for cells $10 \mu \mathrm{m}$ thick or less.

${ }_{10}$ We have been able to study the same region of the CLC cell after cycling into the isotropic and back into the CLC phase (Fig. 6). This procedure leads to the formation of the double linear defect that spans the horizontal diameter, parallel to the imposed farfield orientation, and is pinned on the boojum in the domain's 15 center and at two diametrically opposite points on the periphery of the domain. Vertical cross sections across the linear defect (PQ and OR segments in Fig. 6) reveal that these structures are edge dislocations of Burgers vector $b=p / 2(\mathrm{p} \sim 4 \mu \mathrm{m})$, located nearby the LB-coated surface. The core of the dislocation, which is 20 attached to the LB-coated surface, is split into a pair of $\tau^{-1 / 2} \lambda^{+1 / 2}$ disclinations: defect lines with a combination of singular $(\tau)$ and non-singular $(\lambda)$ cores. Near the center of the domain, the dislocation is connected to the surface by a screw dislocation (see cross section data along the OPQRO circuit in Fig. 6). The

25 distortion caused in the bulk by this dislocation has a much lower penetration length when compared to the edge dislocation in the single-arch configuration (Fig. 5). The two edge dislocations are brought back towards the LB-coated plate by kinks along the disclination, and this generates a kin-antikink pair along the 30 domain boundary, as seen from the series of $\mathrm{XZ}$ and $\mathrm{YZ}$ cross sections in Fig. 6.

\section{Conclusions}

In this work we have studied the topological defects induced in a confined cholesteric liquid crystal with planar far-field alignment 35 by the contact with ordered surfactant monolayer domains. The tilt-orientational order present in the submillimeter monolayer domains propagates into the bulk mesogen, leading to the formation of either single non-singular-core or double singularcore dislocations of the CLC director. As demonstrated with the 40 use of multimodal three-dimensional imaging techniques, the disclinations are subtended between a screw dislocation pinned on a boojum at the center of the domains and glide at the domain boundary leading to the formation of kinks and antikinks. The intrinsic lamellar structure of the CLC sets an additional length

45 scale that determines the penetration depth of the distortion introduced into the bulk mesogen by contact with a monolayer with surface defects. The structure of the defects in the CLC can be understood taking into account the coupling between the orientational chirality of the monolayer domains and the intrinsic 50 chirality of the CLC, as shown with experiments using compensated CLC mixtures featuring a strong temperature variation of the pitch.

\section{Notes and references}

${ }_{55}{ }^{a}$ Departament de Química Física and Institut de Nanociència $i$ Nanotecnologia (IN $\left.{ }^{2} U B\right)$, Universitat de Barcelona, Martí i Franquès 1, 08028 Barcelona, Catalonia, Spain. Fax: +34 934021231; Tel: +34 934021220; E-mail: jignes@ub.edu

${ }^{b}$ Department of Physics and Liquid Crystal Materials Research Center, 60 University of Colorado, Boulder, CO 80309, USA; Fax: 1-303-492-9288; Tel: 1-303-492-7277; E-mail: ivan.smalyukh@colorado.edu

${ }^{c}$ Department of Electrical, Computer, and Energy Engineering and Materials Science and Engineering Program, University of Colorado, Boulder, CO 80309, USA; Renewable and Sustainable Energy Institute, 65 National Renewable Energy Laboratory and University of Colorado, Boulder, CO 80309, USA.

$\dagger$ Electronic Supplementary Information (ESI) available: [details of any supplementary information available should be included here]. See DOI: $10.1039 / \mathrm{b} 000000 \mathrm{x} /$

70

\section{Acknowledgments}

This work has been partially supported by MICINN (Projects FIS2006-03525 and FIS2010-21924-C02-01), DURSI (Project 2009 SGR 1055), and by the NSF Grants No. DMR-0820579 75 (I.I.S.), No. DMR-0844115 (R.T., I.I.S.), and No. DMR- 0847782 (I.I.S.). N. P.-G. acknowledges support from MICINN (Grant No. AP2007-01103 and a travel grant). We thank Noel Clark and Leo Radzihovsky for discussions and Taewoo Lee for help with imaging experiments. We thank Dan Schwartz for the use of LB 80 coating facilities.

\section{SUPPLEMENTARY INFORMATION}

Video sequences with showing changes in the CLC cells in the 85 vicinity of the circular domains during changes in the cholesteric pitch in a compensated mixture. We include both a sequence for domains with the two-point defects and the three-point defect configurations.

90

\section{REFERENCES}

1. P. Oswald and P. Pieranski, Nematic and cholesteric liquid crystals : $95 \quad$ concepts and physical properties illustrated by experiments, Taylor \& Francis, Boca Raton, 2005.

2. V. Kaganer, H. Möhwald and P. Dutta, Reviews of Modern Physics, 1999, 71, 779-819.

3. J. Dreier, J. Brewer and A. C. Simonsen, Soft Matter, 2012, 8, 4894. 100 4. M. Kléman and O. D. Lavrentovich, Soft matter physics : an introduction, Springer, New York, 2003.

5. Q. Liu, B. Senyuk, M. Tasinkevych and Smalyukh, II, Proceedings of the National Academy of Sciences of the United States of America, 2013, 110, 9231-9236.

105 6. E. Pairam, J. Vallamkondu, V. Koning, B. C. van Zuiden, P. W. Ellis, M. A. Bates, V. Vitelli and A. Fernandez-Nieves, Proceedings of the National Academy of Sciences of the United States of America, 2013, 110, 9295-9300.

7. M. E. McConney, A. Martinez, V. P. Tondiglia, K. M. Lee, D. 110 Langley, Smalyukh, II and T. J. White, Adv Mater, 2013, 25, 5880-5885

8. B. Senyuk, Q. Liu, S. He, R. D. Kamien, R. B. Kusner, T. C. Lubensky and Smalyukh, II, Nature, 2013, 493, 200-205. 
9. T. Ohzono, J.-i. Fukuda, K. Suzuki and T. Yamaguchi, Physical Review E, 2012, 86, 030701.

$10 . \quad$ P. Poulin, H. Stark, T. C. Lubensky and D. A. Weitz, Science, 1997, 275, 1770-1773.

5 11. M. Zapotocky, L. Ramos, P. Poulin, T. C. Lubensky and D. A. Weitz, Science, 1999, 283, 209-212.

12. J. C. Loudet, P. Barois and P. Poulin, Nature, 2000, 407, 611613.

13. T. Lopez-Leon, V. Koning, K. B. S. Devaiah, V. Vitelli and A. 10 Fernandez-Nieves, Nature Physics, 2011, 7, 391-394.

14. Y. Choi, T. Atherton, S. Ferjani, R. G. Petschek and C. Rosenblatt, Physical Review E, 2009, 80, 060701.

15. J. H. Kim, M. Yoneya and H. Yokoyama, Nature, 2002, 420, 159-162.

$1516 . \quad$ J.-i. Niitsuma, M. Yoneya and H. Yokoyama, Journal of Applied Physics, 2012, 111, 103507.

17. M. Cavallaro, Jr., L. Botto, E. P. Lewandowski, M. Wang and K. J. Stebe, Proceedings of the National Academy of Sciences of the United States of America, 2011, 108, 20923-20928.

$2018 . \quad$ J. Y. Fang, U. Gehlert, R. Shashidar and C. M. Knobler, Langmuir, 1999, 15, 297-299.

19. M. C. Petty, Langmuir-Blodgett films : an introduction, Cambridge University Press, Cambridge ; New York, 1996.

20. N. Petit-Garrido, R. P. Trivedi, J. Ignes-Mullol, J. Claret, C.

25 Lapointe, F. Sagues and I. I. Smalyukh, Phys Rev Lett, 2011, 107, 177801.

21. N. Petit-Garrido, J. Claret, J. Ignes-Mullol and F. Sagues, Nature communications, 2012, 3, 1001.

22. W. Iglesias, T. J. Smith, P. B. Basnet, S. R. Stefanovic, C. 30 Tschierske, D. J. Lacks, A. Jákli and E. K. Mann, Soft Matter, 2011, 7, 9043.

23. A. Dequidt and P. Oswald, Europhysics Letters (EPL), 2007, 80, 26001.

24. C. A. Schneider, W. S. Rasband and K. W. Eliceiri, Nature

35 Methods, 2012, 9, 671-675.

25. F. Sagues, R. Albalat, R. Reigada, J. Crusats, J. Ignes-Mullol and J. Claret, Journal of the American Chemical Society, 2005, 127, 5296-5297.

26. R. P. Trivedi, T. Lee, K. A. Bertness and I. I. Smalyukh, $40 \quad$ Optics Express, 2010, 18, 27658.

27. Guillamat,Sagués, Ignés, Preprint (ArXiV... 2013)

28. M. Stalder and M. Schadt, Optics Letters, 1996, 21, 19481950 .

$29 . \quad$ S.-W. Suh, K. Joseph, G. Cohen, J. S. Patel and S.-D. Lee, $45 \quad$ Applied Physics Letters, 1997, 70, 2547.

30. I. Smalyukh and O. Lavrentovich, Physical Review E, 2002, 66. 